\title{
Effects of exercise training on quality of life, symptoms of depression, symptoms of anxiety and emotional well-being in type 2 diabetes mellitus: a systematic review
}

\author{
M. M. P. van der Heijden • F. E. P. van Dooren • \\ V. J. M. Pop • F. Pouwer
}

Received: 23 November 2012 / Accepted: 6 February 2013 /Published online: 23 March 2013

(C) Springer-Verlag Berlin Heidelberg 2013

\begin{abstract}
Aims/hypothesis Psychological problems are relatively common in people with type 2 diabetes. It is unclear whether exercise training exerts an effect on quality of life, symptoms of depression, symptoms of anxiety and emotional well-being in people with type 2 diabetes. The aim of this study was to conduct a systematic review to assess the effects of exercise training on these outcomes in people with type 2 diabetes.

Methods MEDLINE, PsycINFO, Embase and ClinicalTrials.gov databases were searched. The review included randomised controlled trials (RCTs) of at least 4 weeks' duration in people with type 2 diabetes that evaluated the effect of exercise training on quality of life, symptoms of depression, symptoms of anxiety and/or emotional wellbeing compared with usual care.

Results Of 1,261 retrieved articles, 20 RCTs were included with a total of 1,719 participants. Quality of life was assessed in 16 studies. Between-group comparisons showed no significant results for aerobic training with the exception of one study, and mixed results for resistance and combined
\end{abstract}

Electronic supplementary material The online version of this article (doi:10.1007/s00125-013-2871-7) contains peer-reviewed but unedited supplementary material, which is available to authorised users.

M. M. P. van der Heijden · F. E. P. van Dooren • V. J. M. Pop •

F. Pouwer $(\bowtie)$

Department of Medical and Clinical Psychology, Center of

Research on Psychology in Somatic Diseases, Tilburg University,

P.O. Box 90153, 5000 LE, Tilburg, the Netherlands

e-mail: f.pouwer@tilburguniversity.edu

F. E. P. van Dooren

Alzheimer Centre Limburg, School for Mental Health and

Neuroscience, Maastricht University, Maastricht, the Netherlands

F. E. P. van Dooren

Maastricht University Medical Center, Department of Medicine,

Maastricht University, Maastricht, the Netherlands training. Symptoms of depression were assessed in four studies. In only one study did the intervention decrease symptoms of depression. Emotional well-being was evaluated in four studies, which also showed conflicting results. Symptoms of anxiety were evaluated in one study, which showed a significant improvement.

Conclusions/interpretation The effects of exercise training on psychological outcomes in people with type 2 diabetes are conflicting. Therefore, there is a need for further highquality RCTs in order to gain greater insight into the role of exercise training in people with type 2 diabetes.

Keywords Anxiety $\cdot$ Depression $\cdot$ Exercise/physical activity $\cdot$ Quality of life $\cdot$ Systematic review $\cdot$ Type 2 diabetes $\cdot$ Well-being

\begin{tabular}{|c|c|}
\hline Abbreviations & \\
\hline CES-D & $\begin{array}{l}\text { Center for Epidemiologic Depression } \\
\text { Scale }\end{array}$ \\
\hline EuroQoL & European Quality of Life questionnaire \\
\hline $\mathrm{RCT}$ & Randomised controlled trial \\
\hline SF12 & 12-item Short-Form Health Survey \\
\hline SF36 & 36-item Short-Form Health Survey \\
\hline SMD & Standardised mean difference \\
\hline WHOQol-BREF & World Health Organization \\
\hline & $\begin{array}{l}\text { Quality of Life questionnaire } \\
\text { (abbreviated version) }\end{array}$ \\
\hline
\end{tabular}

\section{Introduction}

Physical inactivity is one of the major risk factors for the development of type 2 diabetes and its complications [1]. Therefore, an important goal in type 2 diabetes management is achieving and/or maintaining an appropriate level of 
physical activity [2]. One of the mechanisms responsible for the positive effect of physical activity is improved glycaemic control [3]. In addition, appropriate levels of physical activity are associated with a reduced risk of total and cardiovascular mortality $[4,5]$.

Previous systematic reviews and meta-analyses regarding the effects of physical activity in type 2 diabetes have mainly focused on biomedical outcomes such as glycaemic control, body mass and blood pressure [3, 6-8]. Type 2 diabetes is, however, also associated with psychological problems. For example, the prevalence of depression is elevated in people with type 2 diabetes compared with those without diabetes (18\% and $10 \%$, respectively) [9]. People with type 2 diabetes also have a $24 \%$ increased risk of developing depression compared with non-diabetic individuals [10]. Furthermore, compared with the general population, people with diabetes tend to report poorer quality of life [11] and a higher prevalence of general anxiety disorder (14\%) [12]. In addition, cross-sectional studies in people with type 2 diabetes have shown that higher levels of physical activity are associated with fewer symptoms of depression [13] and improved quality of life [14-16].

Results from systematic reviews of intervention studies in healthy and clinical samples showed that exercise training can be used to improve not only biomedical but also psychological outcomes. For example, a meta-analysis of 56 trials showed that in both healthy and rehabilitation groups there was a small but significant improvement in quality of life following exercise training [17]. In two systematic reviews and one metaanalysis that studied the effects of physical activity interventions on symptoms of depression in elderly people, it was found that increased levels of physical activity can lower depression rates and reduce symptoms of depression in the short term [18-20]. Also, a meta-analysis on the effects of physical activity interventions in healthy adults showed that some interventions can decrease symptoms of anxiety [21].

To the best of our knowledge, only one (narrative) review [22] has been conducted to investigate whether exercise training has beneficial effects on quality of life and/or (emotional) well-being in people with type 2 diabetes. The aim of this study was therefore to conduct a systematic review of randomised controlled trials (RCTs) in order to assess the effects of exercise training on quality of life, symptoms of depression, symptoms of anxiety and emotional well-being in people with type 2 diabetes.

\section{Methods}

Search strategy

MEDLINE (PubMed), PsycINFO, Embase and ClinicalTrials.gov databases were electronically searched to the end of March 2012. The search comprised the terms 'randomised controlled trial', 'type 2 diabetes mellitus', 'exercise', 'quality of life', 'depression', 'well-being', 'anxiety' and related entry terms (electronic supplementary material [ESM] Methods). We used a sensitive search strategy for RCTs [23, 24]. In addition, reference lists of identified articles and related reviews were searched manually. Two raters (MMPH and FEPD) independently reviewed titles, abstracts and entire texts for potentially relevant articles. Disagreements were resolved by consensus or by a third rater (FP). Agreement between raters was assessed using Cohen's kappa (к).

\section{Eligibility criteria}

The pre-specified inclusion criteria applied were as follows: (1) RCTs comparing exercise training with usual care, sham exercise or a minimal educational intervention (exercise training was defined as planned, structured and repetitive bodily movement with the intention to improve or maintain one or more components of physical fitness [25]); (2) exercise was prescribed for at least 4 weeks; (3) participants were adults $(\geq 18$ years $)$ and classified as having type 2 diabetes; and (4) the study included assessment of quality of life and/or symptoms of depression and/or symptoms of anxiety, and/or emotional well-being. There was no language restriction or restriction on the year of publication. However, studies in which exercise training was part of an intervention with multiple components (e.g. combined with a diet intervention) were excluded.

\section{Data extraction and analysis}

Data on sample characteristics, intervention characteristics and study results were extracted. Home-based exercise interventions were considered 'individual' interventions. Exercise training was divided into three modes: aerobic (training to improve the efficiency of aerobic energy-producing systems [25]); resistance (training to improve strength, power and muscle endurance [25]); and a combination of both. One investigator (MMPH) performed the data extraction, which was checked by a second investigator (FEPD). If possible, standardised mean differences (SMDs) were calculated. SMDs of 0.2 were considered a small effect, 0.5 a moderate effect and 0.8 a large effect [26]. Positive signs reflect better outcomes, i.e. better quality of life, fewer symptoms of depression, fewer symptoms of anxiety and greater emotional well-being in the intervention group compared with the control group. Because of the heterogeneity of exercise training and outcome measurements, data pooling was not possible.

Assessment of risk of bias

The risk of bias was assessed in accordance with the Preferred Reporting Items for Systematic Reviews and 
Meta-Analyses (PRISMA) statement [27]. The assessment included adequate sequence generation, allocation concealment, blinding of outcome assessors (related to outcomes addressed in the current systematic review), description of losses and exclusions, and use of intention-to-treat analysis. A study was considered to use intention-to-treat analysis when participants were analysed in the group to which they were randomised regardless of the intervention they actually received and when there were no missing data or when appropriate imputation of missing data was performed. Two raters (MMPH and FEPD) independently assessed the risk of bias. Disagreements were resolved by consensus or by a third rater $(\mathrm{FP})$.

\section{Results}

\section{Description of studies}

Overall, 1,261 articles were retrieved from electronic databases and manual searches, 161 articles were included based on review of the title, 33 based on review of the abstract, and 20 [28-47] based on review of the full text article (ESM Fig. 1). The $K$ agreement between raters for article selection was 0.78 .

A total of 1,719 people with type 2 diabetes were included in the studies. Samples sizes ranged from 18 [30] to 84 [32] participants, with the exception of Reid et al [44] $(n=218)$ and Nicolucci et al [42] $(n=606)$. The mean age of the participants ranged from 43 years [30] to 70 years [46]. The mean BMI ranged from $25 \mathrm{~kg} / \mathrm{m}^{2}$ [28] to $40 \mathrm{~kg} / \mathrm{m}^{2}$ [31], mean $\mathrm{HbA}_{1 \mathrm{c}}$ ranged from $6.4 \%(46 \mathrm{mmol} / \mathrm{mol}$ ) [32] to $9.0 \%$ (75 mmol/mol) [37] (Table 1). In none of the studies was low quality of life or low emotional well-being, or suffering from symptoms of depression or anxiety, an inclusion criterion.

Four studies compared two or three modes of exercise training with a control group [28, 34, 38, 44]. Including these studies, a total of ten studies examined the effect of aerobic training [28, 30, 33-35, 38, 39, 41, 44, 45], five the effect of resistance training $[28,31,40,43,44]$, and ten the effect of combined training [29, 32, 34, 36-38, 42, 44, 46, 47]. In studies examining aerobic training, (Nordic) walking was the most common exercise training [28, 33-35, 45]. Other forms of aerobic training were treadmills and/or bicycle ergometers [44] and circuit training [38]. In three studies, the specific form of aerobic training was not specified [30, $39,41]$. Studies examining resistance training all followed a progressive schedule, using pneumatic/weight resistance training machines [40, 44], multigym apparatus/dumb-bells $[28,43]$ or exercise bands [31]. Combined training consisted of t'ai chi $[37,47]$ or a combination of aerobic (e.g. walking, treadmills, outdoor activities) and resistance training (e.g. circuit training, weight machines) $[29,32,34,36,38,42$, $44,46]$. In one study the aerobic training [33] and in two studies the combined training $[32,36]$ was not supervised (Table 1).

The duration of the training sessions varied from $10 \mathrm{~min}$ $[29,36]$ to $60 \mathrm{~min}[35,37,39]$. The frequency of the training sessions varied from once a week $[34,37]$ to (at least) five times a week $[31,36,41,45]$. The duration of the total training period varied from 6 weeks [39] to 12 months [42] (Table 1).

Adherence rates were reported in 14 studies [29, 31-36, $38,41-44,46,47]$. Dropouts were (partially) reported in all but one study [40]. None of the studies had a dropout rate $>20 \%$ (Table 1).

Outcomes

Quality of life Six studies examined the effect of aerobic training on quality of life [30, 33-35, 38, 44]. Adequate random sequence generation was performed in four studies $[30,33,38,44]$, adequate allocation concealment in three studies [30, 33, 44], and blinding of outcome assessment in two studies $[38,44]$. All studies gave an adequate description of losses and exclusions [30, 33-35, 38, 44]. An intention-to-treat analysis was performed in four studies [30, 33, 34, 44] (ESM Table 1).

Three studies used the 36-item Short-Form Health Survey (SF36) [34, 38, 44], one the abbreviated version of the World Health Organization Quality of Life questionnaire (WHOQol-BREF) [30], one the Swedish Health-Related Quality of Life questionnaire (SWED-QUAL) [33], and one the Quality of Well-Being scale [35]. Reid et al [44] did not perform a statistical test to compare the mean scores on the SF36 mental component scale of the participants who received supervised aerobic training and those who received usual care (respectively, baseline 52.4 \pm 9.3 and $47.3 \pm 9.6$, post intervention $54.5 \pm 9.6$ and $54.0 \pm 9.6$; SMD 0.05; $n=103$ ). The same was true for the SF36 physical component scale (respectively, baseline 49.7 \pm 7.2 and 49.2 \pm 7.4 , post intervention $49.9 \pm 7.5$ and $48.8 \pm 7.5$; SMD 0.15; $n=103$ ). Four smaller studies (sample sizes between 18 [30] and 44 [34]) reported no significant effects of aerobic training on quality of life compared with usual care [30, 34, 38] or education [35] immediately post intervention. SMDs could be computed for two of these studies varying from -0.68 ('general health' SF36 subscale) [38] to 0.69 [30]. Two studies performed an additional follow-up measurement. Gram et al [34] evaluated Nordic walking during 4 months with follow-up after 1 year. Kaplan et al [35] also evaluated walking during 10 weeks with follow-up after 6 , 12 and 18 months. Neither found any significant effects of exercise. However, Fritz et al [33] also evaluated a 4 month Nordic walking intervention $(n=50)$ and found significant 


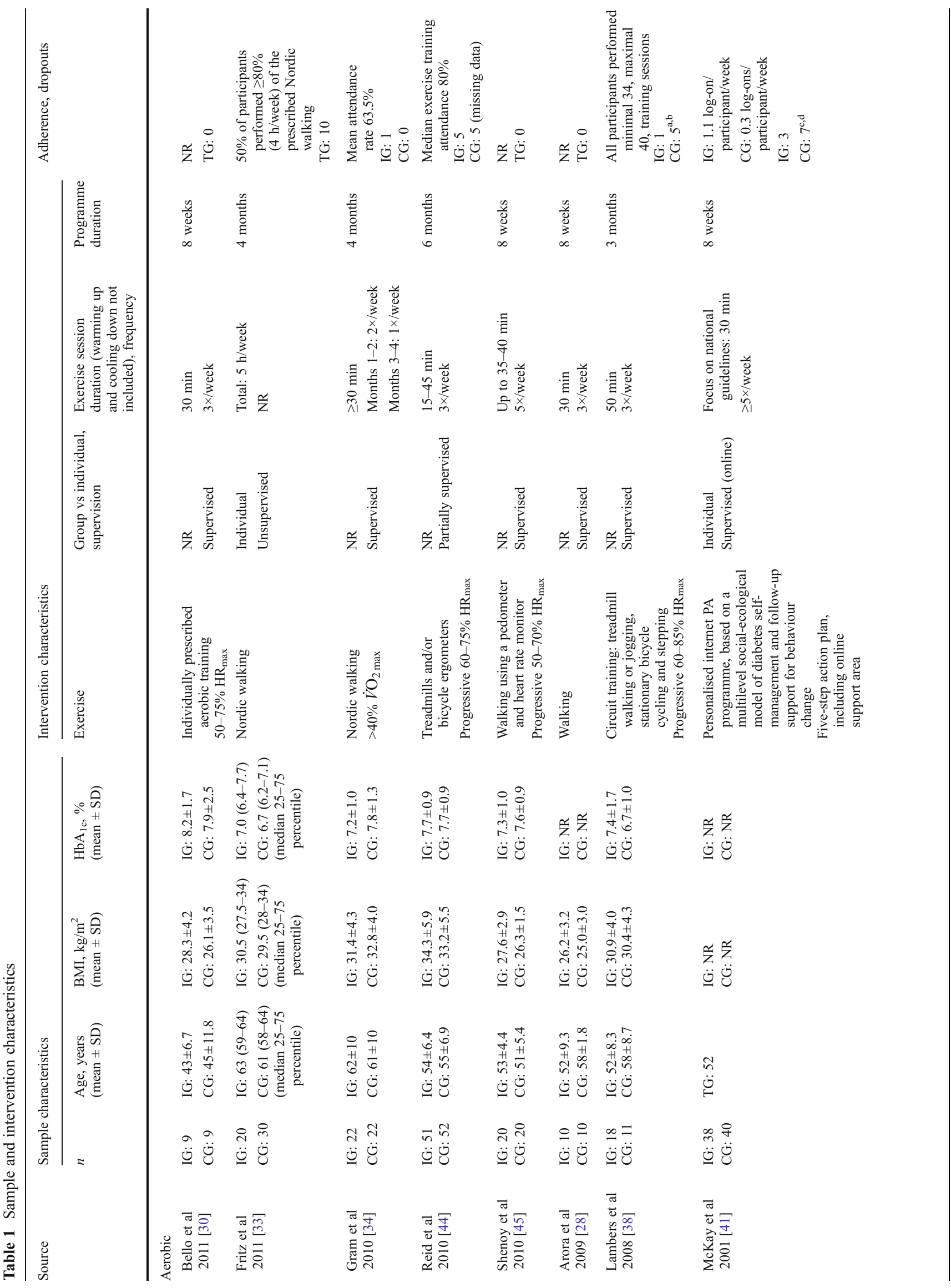




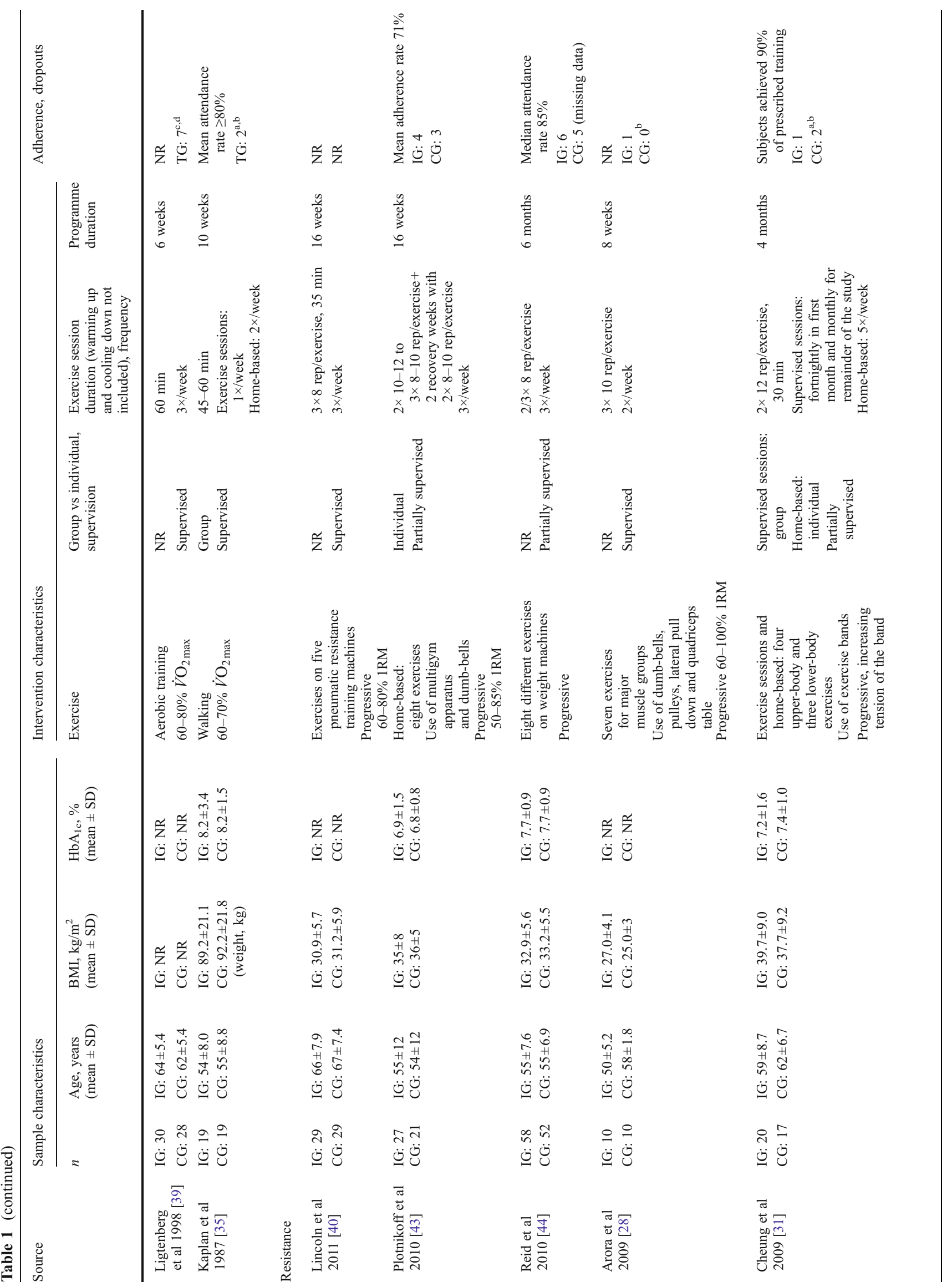




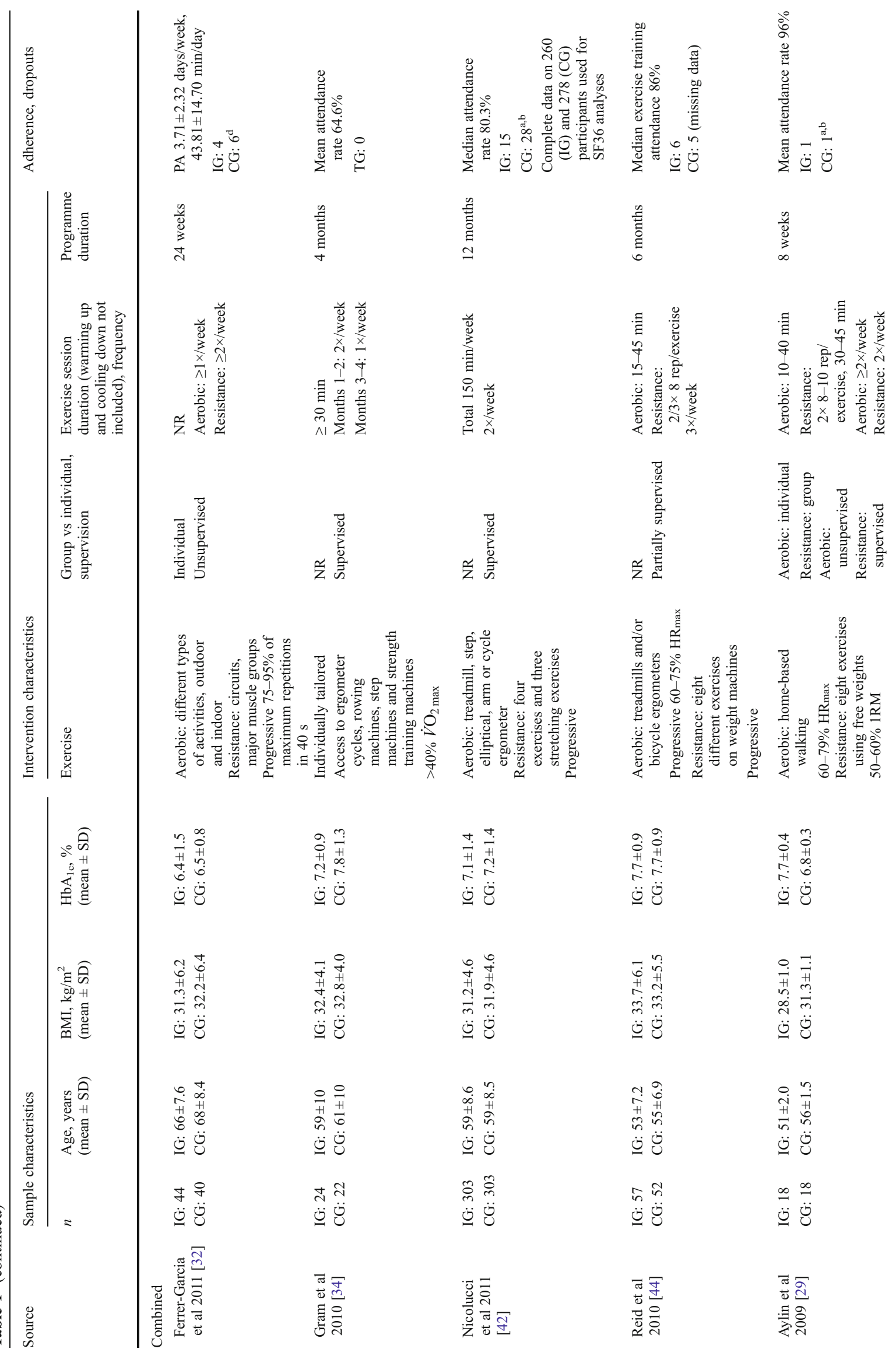




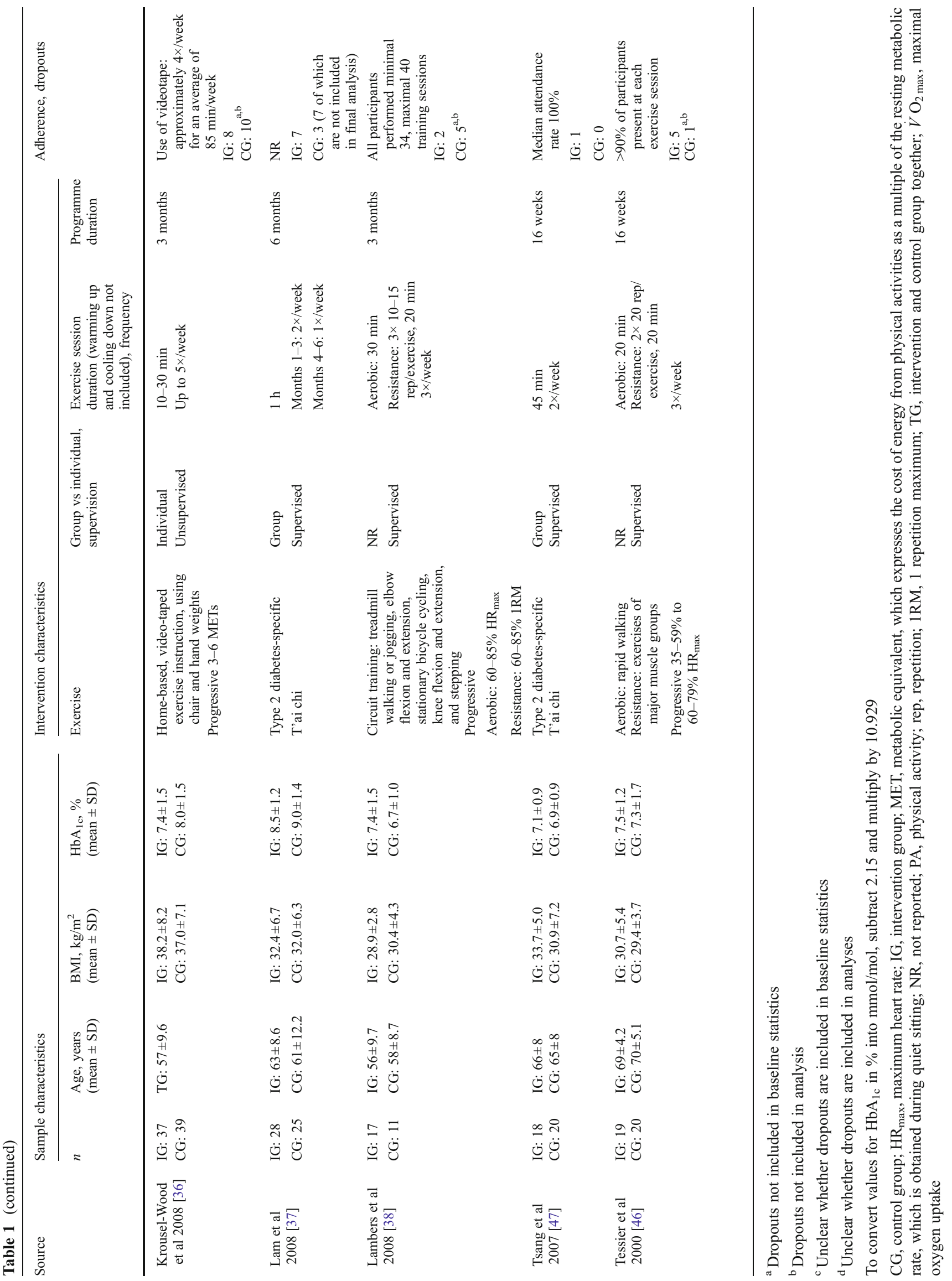


positive effects compared with usual care on two of 13 subscales, i.e. 'satisfaction with physical health' $(p=0.03$, reported effect size 0.7$)$ and 'sleep' ( $p=0.03$, reported effect size 0.5 ) (Table 2).

Four studies examined the effect of resistance training on quality of life [31, 40, 43, 44]. Adequate random sequence generation, allocation concealment, blinding of outcome assessment and intention-to-treat analysis were performed in two studies [43, 44]. Three studies gave an adequate description of losses and exclusions [31, 43, 44] (ESM Table 1). Three studies used the SF36 [31, 40, 44] and one used the 12-item Short-Form Health Survey (SF12) [43], all immediately post intervention. Results were mixed for the mental component scale of the SF36/SF12. Lincoln et al [40] examined the effect of 16 weeks' training and found a significant positive effect of resistance training compared with usual care $(p<0.001$; SMD $1.15 ; n=58)$. However, Reid et al [44] reported a significant positive effect favouring usual care $(p<0.001$; SMD $-0.38 ; n=110)$ compared with 6 months' training on exercise machines. Plotnikoff et al [43] found no significant effect of their home-based training (SMD 0.58; $n=48$ ). Results on the physical component scale of the SF36/SF12 were also mixed. Reid et al [44] found no significant effect on the physical component scale (SMD 0.32; $n=110$ ), which is in line with the finding of Plotnikoff et al [43] (SMD 0.12; $n=48$ ). Cheung et al [31] found a significant positive effect of home-based training with exercise bands compared with usual care in their sample with the highest mean BMI (intervention group $39.7 \mathrm{~kg} / \mathrm{m}^{2}$ ), on the subscale 'general health' ( $p=0.02$; SMD of change scores $0.87 ; n=37$ ) (Table 2).

All ten studies examining the effect of combined training assessed quality of life [29, 32, 34, 36-38, 42, 44, 46, 47]. Adequate random sequence generation was performed in five studies [37, 38, 42, 44, 47], adequate allocation concealment in four studies [37, 42, 44, 47], and blinding of outcome assessment in three studies [37, 38, 44]. All studies gave an adequate description of losses and exclusions [29, 32, 34, 36-38, 42, 44, 46, 47]. An intention-to-treat analysis was performed in three studies [34, 44, 47] (ESM Table 1). Eight studies used the SF36 [29, 34, 36-38, 42, 44, 47], one the European Quality of Life questionnaire (EuroQoL) [32], and one a combination of the Diabetes Quality of LifeDiabetes Control and Complications Trial (DCCT) Research Group questionnaire and the Modified Quality of Life Measure for Youths [46]. Results were mixed. Five studies with sample sizes between 28 [38] and 76 [36] participants found no significant effects of combined training on quality of life compared with usual care [34, 36-38, 46]. SMDs could be computed for four of these studies varying from -0.63 ('social function' SF36 subscale) [38] to 0.43 [36]. However, Nicolucci et al [42], who studied the largest sample $(n=606)$ and had the intervention with the longest duration (12 months), found positive effects of intensive combined training on all subscales of the SF36 (SMDs between 0.67 for 'physical functioning' and 0.94 for 'general health'). Aylin et al [29] found significant positive effects of resistance training combined with homebased walking compared with usual care on three of seven subscales of the SF36, i.e. 'emotional role' ( $p=0.03$; SMD $0.24)$, 'mental health' ( $p=0.02$; SMD 0.17$)$ and 'vitality' $(p<0.001$; SMD 0.33), in their sample of 36 participants. Furthermore, Tsang et al [47] found a significant positive effect of 16 weeks of t'ai chi training compared with sham exercise on the only SF36 subscale ('social function') they assessed ( $p=0.04$; SMD $0.13 ; n=38$ ). After correction for percentage of body fat, however, there was no longer a significant effect. Reid et al [44] also found no significant effect on the physical component scale of the SF36 (SMD $0.12 ; n=109$ ). They did, however, find a positive significant effect on the mental component scale favouring usual care $(p<0.001$; SMD -0.17$)$ compared with combined training. Ferrer-Garcia et al [32] only tested for within-subject effects of combined training on quality of life in the sample with the best glycaemic control (intervention group, $\mathrm{HbA}_{1 \mathrm{c}}$ $6.4 \% / 46 \mathrm{mmol} / \mathrm{mol}$ ) in the included studies. They found significant improvements in the combined training group but not in the usual care group (SMD EuroQol 0.49, SMD EuroQol visual analogue scale $0.71 ; n=84$ ). Gram et al [34] were the only group to assess quality of life 8 months post intervention $(n=44)$. They found no significant effect of individually tailored combined training on quality of life compared with usual care (Table 2).

Both Fritz et al [33] and Reid et al [44] noted that baseline quality of life scores of the intervention participants did not differ from those of people without type 2 diabetes. Plotnikoff et al [43] also remarked that quality of life scores were relatively high at baseline compared with those of the general adult population in their province. This phenomenon of the 'healthy volunteer' was also addressed by Tessier et al [46].

Symptoms of depression Four studies examined the effects of exercise training on symptoms of depression [29, 39-41]. Adequate random sequence generation and adequate allocation concealment was performed in one study [41]. It was unclear in all studies whether blinding of outcome assessment was performed [29, 39-41]. One study did not have an adequate description of losses and exclusions [40], and in none of the studies was an intention-to-treat analysis performed or it was unclear whether one was done [29, 39-41] (ESM Table 1).

Two studies tested whether aerobic training affected symptoms of depression [39, 41]. McKay et al [41] assessed the effects of an 8 week internet physical activity intervention on 


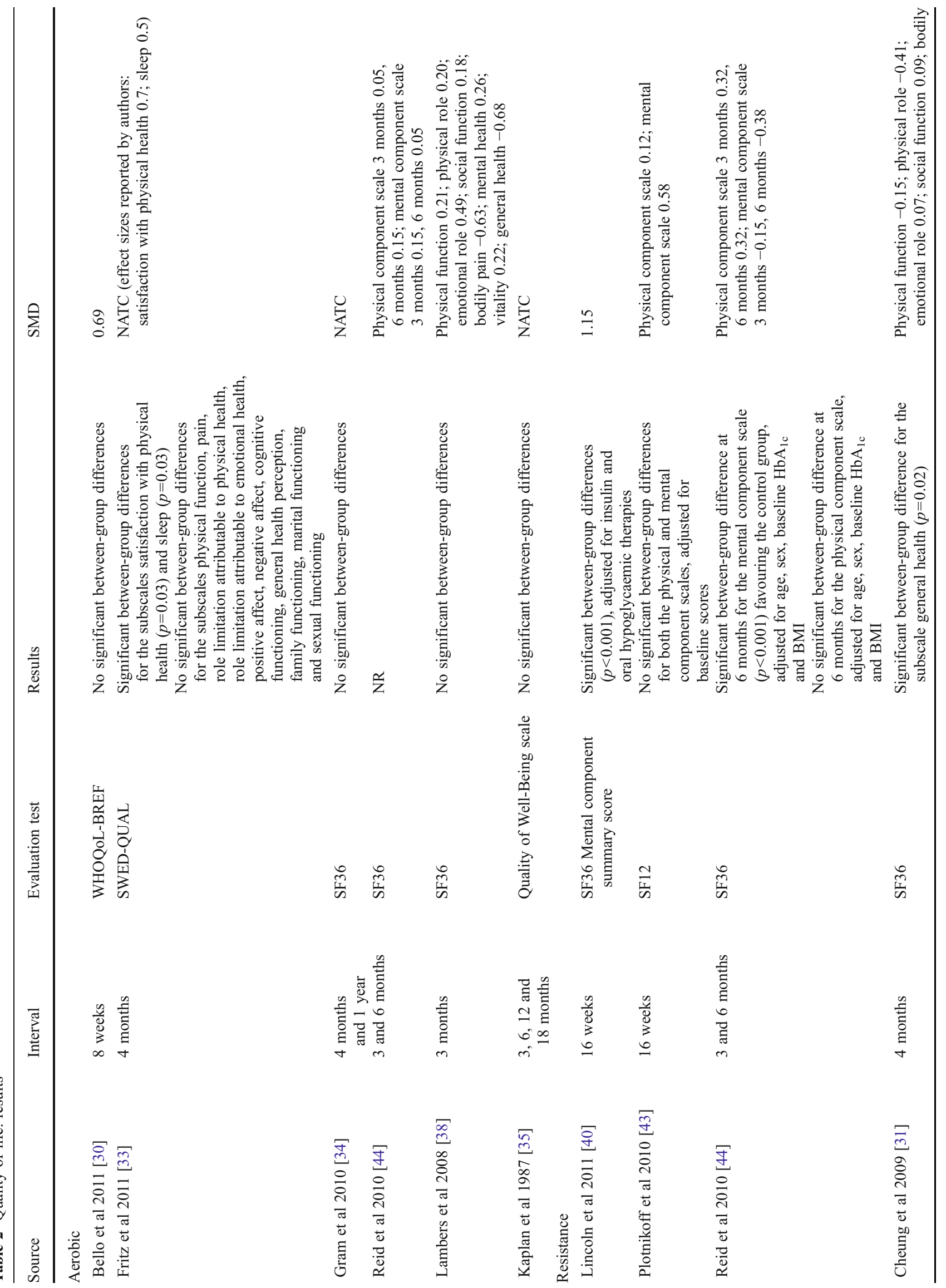




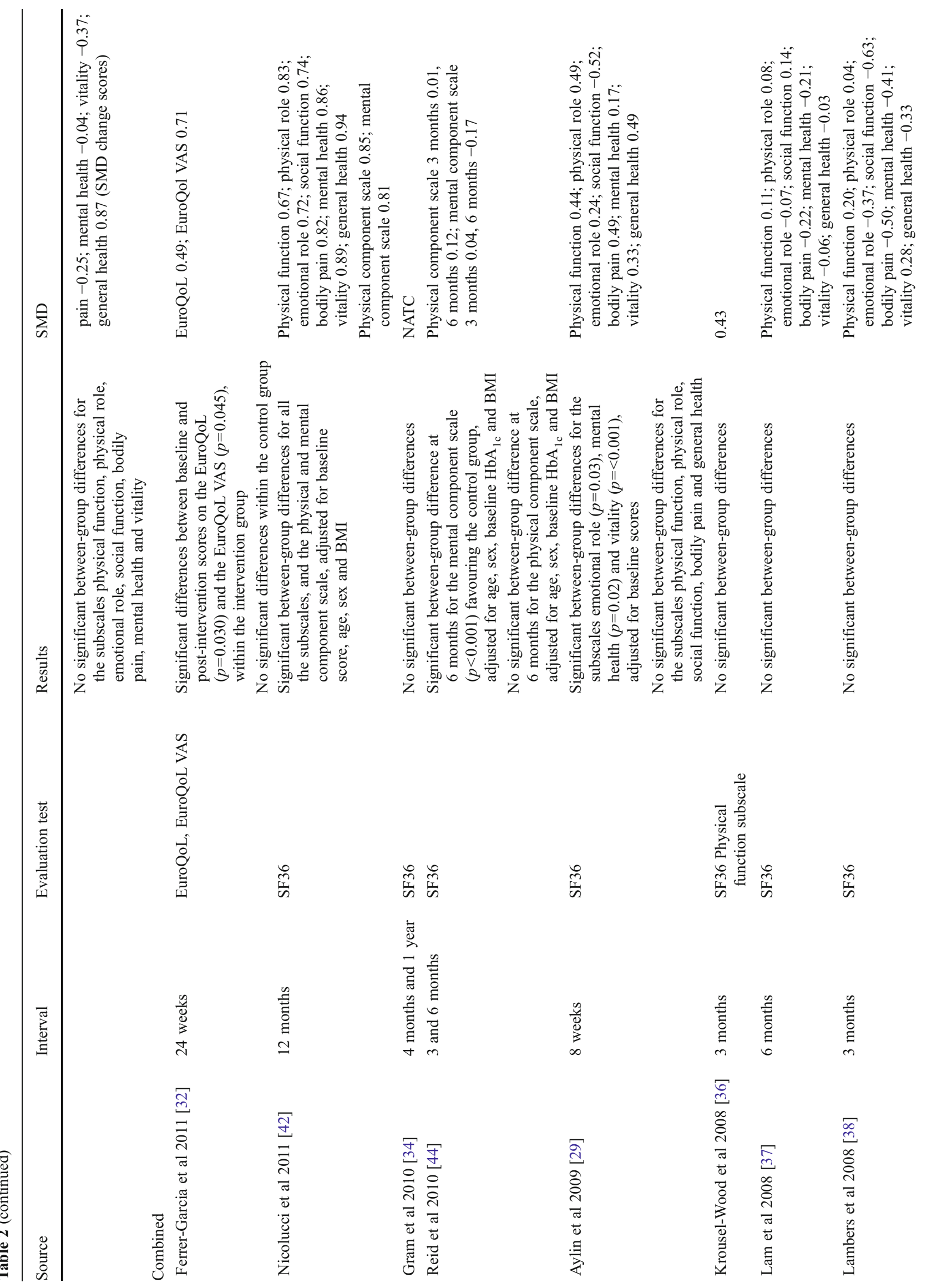




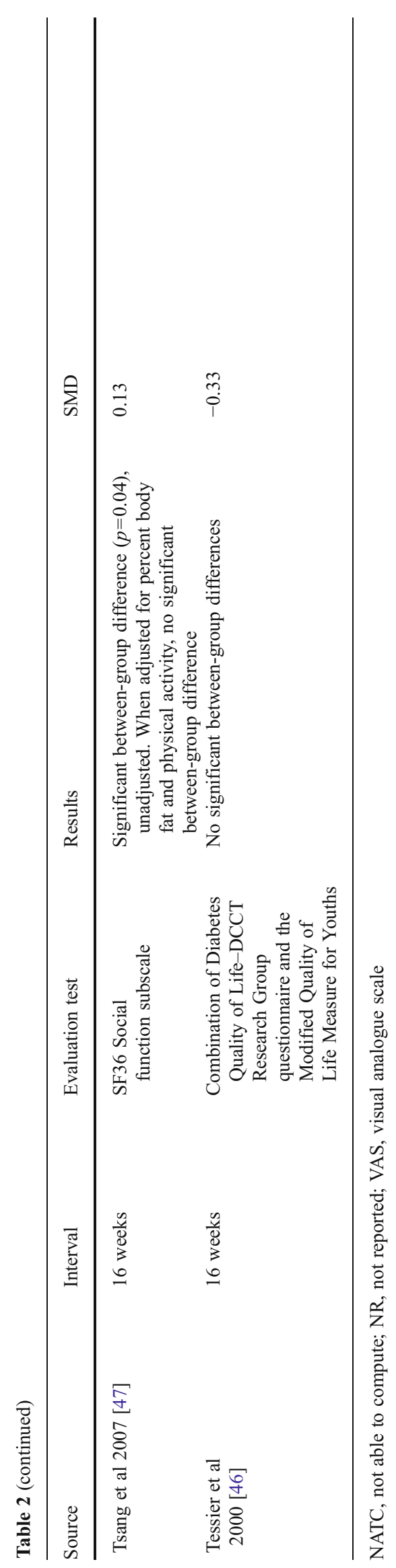

备 Springer 
symptoms of depression, using the ten-item Center for Epidemiologic Depression Scale (CES-D), and found no significant effect (SMD $0.37 ; n=78$ ). Similarly, Ligtenberg et al [39] found no significant positive effect on the 'depression' subscale of the General Well-Being Questionnaire of their 6 weeks' training programme in 58 participants. Lincoln et al [40] examined the effects of training with pneumatic resistance machines on symptoms of depression. They found that the intervention decreased symptoms of depression $(p<0.001$; SMD $1.51 ; n=58)$, assessed with the Geriatric Depression Scale immediately post intervention, compared with usual care. Aylin et al [29] found no significant effect of homebased walking combined with resistance training compared with usual care, assessed with the CES-D immediately post intervention (SMD 0.21; $n=36$ ) (Table 3).

Lincoln et al [40] were the only investigators who reported the number of participants with elevated levels of depressive symptoms: $52 \%$ of their participants had levels of symptoms of depression that were likely to indicate clinically relevant distress at baseline.

Symptoms of anxiety Ligtenberg et al [39] examined the effect of 6 weeks' aerobic training on symptoms of anxiety. They gave an adequate description of losses and exclusions, but for the other domains it was unclear whether there was a high or a low risk of bias (ESM Table 1). These researchers found that aerobic training significantly reduced symptoms of anxiety compared with education ( $p=0.007$; SMD 0.66 ; $n=58$ ), assessed using a subscale of the Well-Being Questionnaire immediately post intervention (Table 4).

Emotional well-being Of the four studies that examined the effects of exercise training on emotional well-being [28, 39, $44,45]$, two performed an adequate random sequence generation and an intention-to-treat analysis [44, 45]. Adequate allocation concealment and blinding of outcome assessment were performed in one study [44]. All studies gave an adequate description of losses and exclusions $[28,39,44$, 45] (ESM Table 1).

All four studies used (different versions of) the General Well-Being Questionnaire, immediately post intervention, and addressed the effects of aerobic training on emotional well-being [28, 39, 44, 45]. Two studies found significant positive effects of aerobic training on emotional well-being $[39,45]$ : Shenoy et al [45] evaluated the effect of 8 weeks of walking using a pedometer compared with usual care $(p<0.001$; SMD 2.26; $n=40)$; and Ligtenberg et al [39] evaluated 6 weeks of aerobic training compared with education ( $p=0.023$; SMD $0.69 ; n=58$ ). However, Arora et al [28] found no significant effect of walking compared with usual care (SMD 0.39; $n=20$ ). Reid et al [44] did not perform a statistical test to compare the mean scores on the Well-Being Questionnaire of the participants who received aerobic training with those who received usual care (respectively, baseline $25.6 \pm 5.2$ and $24.6 \pm 5.3$, post intervention $27.3 \pm 5.3$ and $25.9 \pm 5.4$; SMD 0.26; $n=103$ ). Two studies addressed the effects of resistance training on emotional well-being compared with usual care, immediately post intervention [28, 44]. Reid et al [44] found no significant effect (SMD 0.31; $n=110$ ), while Arora et al [28] found a significant positive effect despite a considerably smaller sample size (SMD 1.33; $n=20$ ). Reid et al [44] also evaluated the effects of combined training compared with usual care on emotional well-being, finding no significant effect (SMD $0.13 ; n=109$ ) immediately post intervention (Table 4).

\section{Discussion}

This is the first systematic review focusing on the effects of exercise training on quality of life, symptoms of depression,

Table 3 Symptoms of depression: results

\begin{tabular}{|c|c|c|c|c|}
\hline Source & Interval & Evaluation test & Results & SMD \\
\hline \multicolumn{5}{|l|}{ Aerobic } \\
\hline McKay et al 2001 [41] & 8 weeks & CES-D & $\begin{array}{l}\text { No significant between-group difference, } \\
\text { adjusted for sex }\end{array}$ & 0.37 \\
\hline Ligtenberg et al 1998 [39] & 6 weeks & $\begin{array}{l}\text { General Well-Being Questionnaire } \\
\text { ( } 22 \text { items), including depression } \\
\text { subscale }\end{array}$ & No significant between-group difference & NATC \\
\hline \multicolumn{5}{|l|}{ Resistance } \\
\hline Lincoln et al 2011 [40] & 16 weeks & Geriatric Depression Scale & $\begin{array}{l}\text { Significant between-group difference }(p<0.001) \text {, } \\
\text { adjusted for insulin and oral hypoglycaemic } \\
\text { therapies }\end{array}$ & 1.51 \\
\hline \multicolumn{5}{|l|}{ Combined } \\
\hline Aylin et al 2009 [29] & 8 weeks & CES-D & No significant between-group difference & 0.21 \\
\hline
\end{tabular}

NATC, not able to compute 


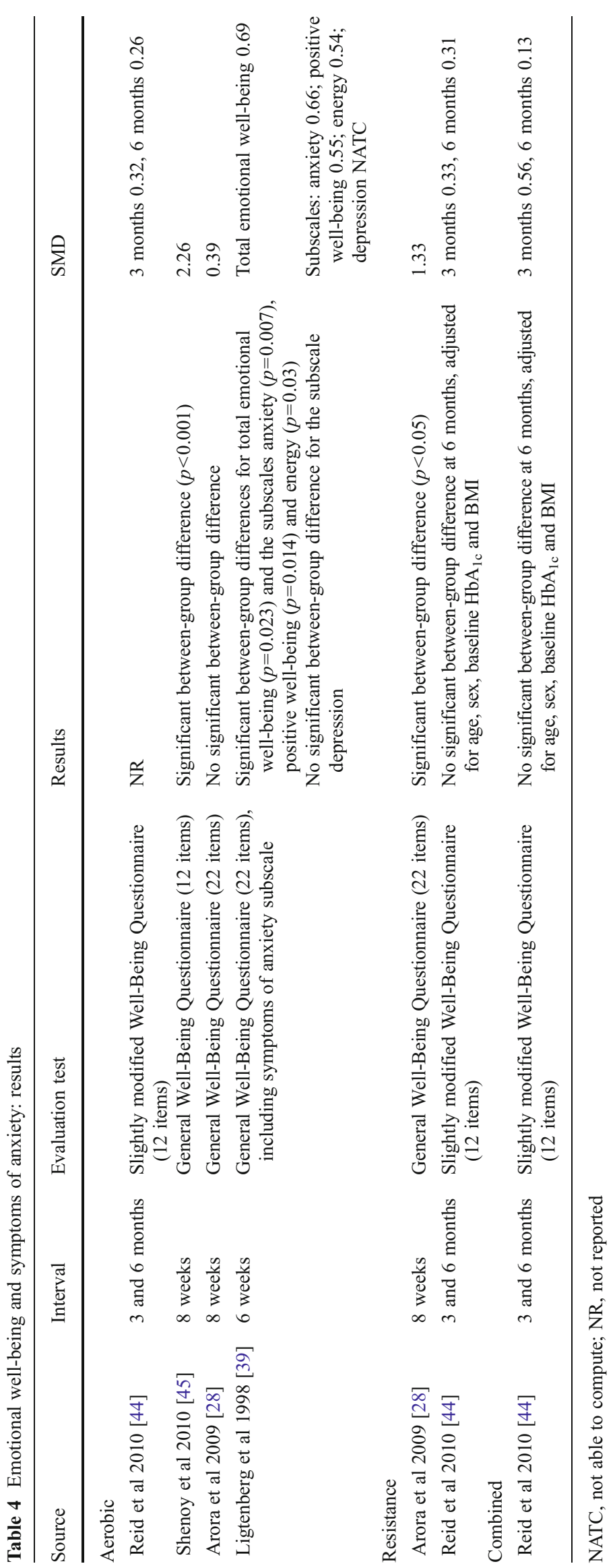


symptoms of anxiety and emotional well-being in people with type 2 diabetes $(n=1,719)$. The 20 included studies were rather heterogeneous in terms of type of intervention, target population, instruments used to measure the outcomes and risk of bias. This heterogeneity was reflected in the mixed results that were found. Aerobic training did not seem to have positive effects on quality of life or symptoms of depression, but a positive effect was found on anxiety (although this was measured in only one study), and effects on emotional well-being were mixed. The effects of resistance training on quality of life and well-being were also mixed. Resistance training reduced symptoms of depression, yet this was assessed in only one study. Studies evaluating combined training found mixed effects on quality of life and no effects on symptoms of depression or emotional well-being. Because of the small number of studies, especially concerning symptoms of anxiety, symptoms of depression and well-being, these results should be interpreted with caution.

A positive effect of exercise training on quality of life in people with type 2 diabetes was expected. In this review, however, the effects of exercise training on quality of life were mixed and were almost absent for aerobic training. There are several explanations for these mixed results. Apart from the heterogeneity of exercise training and participants, there are also different operationalisations of the concept of quality of life. This is reflected in the variety of quality of life subscales that were used in the studies included in the current review. Also, diabetes-specific quality of life might have a stronger association with exercise training than health-related/general quality of life. For example, it was found that a decrease in $\mathrm{HbA}_{1 \mathrm{c}}$, which is related to physical activity [3], was not associated with improved health-related quality of life but was associated with improved diabetesspecific quality of life [48]. However, only one study included in the current review partially assessed diabetesspecific quality of life [46].

In addition, studies included in this review predominantly measured outcomes immediately post intervention. Quality of life is, however, adversely associated with complications of diabetes $[49,50]$ but is not associated with short-term diabetic control [50], both of which are related to physical activity $[1,3]$. Therefore, the expected positive effect of exercise training on quality of life might be more prominent in the long term. It should, however, be mentioned that the required long-term compliance with exercise is still of major concern [51].

One study that was included in the current review, with $52 \%$ of the participants having clinically relevant levels of depressive symptoms, found positive effects of exercise training [40]. This is in line with earlier reported positive effects of exercise training in depressed elderly people [20] and depressed adults [52]. However, three studies in the current review that did not report on the number of participants with clinical depression, nor had it as an inclusion criterion, found no positive effect. This might suggest that exercise training could have a positive effect on clinical depression and clinically meaningful levels of depressive symptoms, but not on subclinical levels of depression. The mechanisms that might link exercise to improvements in (symptoms of) depression are still uncertain. A few of the proposed mechanisms for the relationship between exercise and depression are via improvement of mood as a result of improved fitness [52], enhanced self-efficacy and the release of endorphins [53].

As anxiety can influence daily life to a large extent, it is important to investigate possible treatment modalities. However, symptoms of anxiety were assessed and reported in only one of the included studies [39]. Although the positive results of that study are in line with results of a review on the effects of physical activity in healthy people with symptoms of anxiety [21], no conclusion can be drawn based on only one study.

Four of five studies that assessed emotional well-being showed positive effects of exercise training. Because of the limited number of studies addressing emotional well-being, this finding should be interpreted with caution. In addition, people with type 2 diabetes face specific diabetes-related problems that might influence their well-being. The use of a diabetes-specific well-being questionnaire might lead to different results. However, no study using such a questionnaire was included.

Floor and ceiling effects can occur when there is little room for improvement due to very low or very high scores at baseline. This might be a partial explanation for the lack of clear effects of exercise training on psychological outcomes in this review. In none of the studies was it an inclusion criterion to have low quality of life or emotional well-being, or to suffer from symptoms of depression or anxiety. In addition, 'the healthy volunteer' [46] might be more willingly to participate in an exercise intervention. For example, having symptoms of depression predicts subsequent non-adherence to self-care, such as exercise, in patients with type 2 diabetes [54]. In several of the included studies this issue was addressed as an explanation for a lack of positive effects [33, 43, 44, 46]. Lincoln et al [40] were the only investigators to find a positive effect on symptoms of depression, but they were also the only ones who reported that $52 \%$ of their participants had levels of clinically relevant symptoms of depression at baseline.

There are several limitations that restricted us in drawing conclusions. We were not able to retrieve a sufficient number of homogenous studies, as a consequence of which it was decided that statistical pooling by means of a metaanalysis was not feasible. However, in order to make some comparisons, we divided the studies into three modes of exercise: aerobic, resistance and combined exercise. Furthermore, only a few studies addressed symptoms of 
depression, symptoms of anxiety or emotional well-being. Although there appears to be increasing attention to the psychological effects of physical activity in type 2 diabetes, as reflected by the fact that the majority of the included studies were published in the past 5 years, still the main focus of research in type 2 diabetes and physical activity is on biomedical outcomes [3, 6-8]. Also, a substantial number of the studies had a relatively small sample size, resulting in a possible lack of power to detect significant changes in outcomes. Moreover, as is the case with all systematic reviews, there is a risk of publication bias.

The inconclusive results in this study underline the need for future research into the effects of exercise training on psychological outcomes in people with type 2 diabetes. It is unclear which elements of exercise training might have contributed to positive effects in some of the studies. For example, in this review no answer is given to the question whether physical activity itself or the attention of caregivers and contact with peers might have led to some of the positive findings. This requires more well-described, highquality RCTs (with sufficient power) that also evaluate the level of physical activity; this was done in approximately only half of the included studies. More research on this topic would also enable the performance of a meta-analysis and an assessment of the effects of different modes, intensities and durations of exercise training.

We conclude that this first systematic review of the literature showed that the effects of exercise training on quality of life, symptoms of depression, symptoms of anxiety and emotional well-being in people with type 2 diabetes are conflicting; therefore, more research is warranted.

Duality of interest The authors declare that there is no duality of interest associated with this manuscript.

Contribution statement All authors contributed to the design of the study. MMPH and FEDP performed the article search and data extraction. MMPH drafted the manuscript. All authors reviewed/edited the manuscript. All authors approved the various versions including the final version of the manuscript.

\section{References}

1. Sullivan PW, Morrato EH, Ghushchyan V, Wyatt HR, Hill JO (2005) Obesity, inactivity, and the prevalence of diabetes and diabetes-related cardiovascular comorbidities in the U.S., 20002002. Diabetes Care 28:1599-1603

2. American Diabetes Association (2012) Standards of medical care in diabetes-2012. Diabetes Care 35(Suppl 1):S11-S63

3. Umpierre D, Ribeiro PAB, Kramer CK (2011) Physical activity advice only or structured exercise training and association with
HbAlc levels in type 2 diabetes. A systematic review and metaanalysis. JAMA 305:1790-1799

4. Hu G, Jousilahti P, Barengo NC, Qiao Q, Lakka TA, Tuomilehto J (2005) Physical activity, cardiovascular risk factors, and mortality among Finnish adults with diabetes. Diabetes Care 28:799-805

5. Lee IM, Shiroma EJ, Lobelo F, Puska P, Blair SN, Katzmarzyk PT (2012) Effect of physical inactivity on major non-communicable diseases worldwide: an analysis of burden of disease and life expectancy. Lancet 380:219-229

6. Chudyk A, Petrella RJ (2011) Effects of exercise on cardiovascular risk factors in type 2 diabetes: a meta-analysis. Diabetes Care 34:1228-1237

7. Conn VS, Hafdahl AR, Mehr DR, LeMaster JW, Brown SA, Nielsen PJ (2007) Metabolic effects of interventions to increase exercise in adults with type 2 diabetes. Diabetologia 50:913-921

8. Snowling NJ, Hopkins WG (2006) Effects of different modes of exercise training on glucose control and risk factors for complications in type 2 diabetic patients: a meta-analysis. Diabetes Care 29:2518-2527

9. Ali S, Stone MA, Peters JL, Davies MJ, Khunti K (2006) The prevalence of co-morbid depression in adults with type 2 diabetes: a systematic review and meta-analysis. Diabet Med 23:1165-1173

10. Nouwen A, Winkley K, Twisk J et al (2010) Type 2 diabetes mellitus as a risk factor for the onset of depression: a systematic review and meta-analysis. Diabetologia 53:2480-2486

11. Rubin RR, Peyrot M (1999) Quality of life and diabetes. Diabetes Metab Res Rev 15:205-218

12. Grigsby AB, Anderson RJ, Freedland KE, Clouse RE, Lustman PJ (2002) Prevalence of anxiety in adults with diabetes: a systematic review. J Psychosom Res 53:1053-1060

13. Lysy Z, Da Costa D, Dasgupta K (2008) The association of physical activity and depression in type 2 diabetes. Diabet Med 25:1133-1141

14. Glasgow RE, Ruggiero L, Eakin EG, Dryfoos J, Chobanian L (1997) Quality of life and associated characteristics in a large national sample of adults with diabetes. Diabetes Care 20:562567

15. Green AJ, Fox KM, Grandy S (2011) Impact of regular exercise and attempted weight loss on quality of life among adults with and without type 2 diabetes mellitus. J Obes doi:10.1155/2011/172073

16. Imayama I, Plotnikoff RC, Courneya KS, Johnson JA (2011) Determinants of quality of life in type 2 diabetes population: the inclusion of personality. Qual Life Res 20:551-558

17. Gillison FB, Skevington SM, Sato A, Standage M, Evangelidou S (2009) The effects of exercise interventions on quality of life in clinical and healthy populations; a meta-analysis. Soc Sci Med 68:1700-1710

18. Blake H, Mo P, Malik S, Thomas S (2009) How effective are physical activity interventions for alleviating depressive symptoms in older people? A systematic review. Clin Rehabil 23:873-887

19. Sjosten N, Kivela SL (2006) The effects of physical exercise on depressive symptoms among the aged: a systematic review. Int $\mathrm{J}$ Geriatr Psychiatr 21:410-418

20. Bridle C, Spanjers K, Patel S, Atherton NM, Lamb SE (2012) Effect of exercise on depression severity in older people: systematic review and meta-analysis of randomised controlled trials. Br J Psychiatry 201:180-185

21. Conn VS (2010) Anxiety outcomes after physical activity interventions: meta-analysis findings. Nurs Res 59:224-231

22. Zanuso S, Balducci S, Jimenez A (2009) Physical activity, a key factor to quality of life in type 2 diabetic patients. Diabetes Metab Res Rev 25(Suppl 1):S24-S28

23. Robinson KA, Dickersin K (2002) Development of a highly sensitive search strategy for the retrieval of reports of controlled trials using PubMed. Int J Epidemiol 31:150-153 
24. Wong SS, Wilczynski NL, Haynes RB (2006) Developing optimal search strategies for detecting clinically sound treatment studies in Embase. J Med Libr Assoc 94:41-47

25. U.S. Department of Health and Human Services (1996) Physical activity and health: a report of the Surgeon General. Available from www.cdc.gov/nccdphp/sgr/pdf/chap2.pdf, accessed 2 November 2012

26. Higgins JPT, Green S (eds) (2011) Cochrane handbook for systematic reviews of interventions 5.1.0. [updated March 2011]. In: The Cochrane Library. Wiley, Chichester

27. Liberati A, Altman D, Tetzlaff J et al (2009) The PRISMA statement for reporting systematic reviews and meta-analyses of studies that evaluate health care interventions: explanation and elaboration. Ann Intern Med 151:W1-W30

28. Arora E, Shenoy S, Sandhu JS (2009) Effects of resistance training on metabolic profile of adults with type 2 diabetes. Indian J Med Res 129:515-519

29. Aylin K, Arzu D, Sabri S, Handan TE, Ridvan A (2009) The effect of combined resistance and home-based walking exercise in type 2 diabetes patients. Int J Diabetes Dev Ctries 29:159-165

30. Bello AI, Owusu-Boakye E, Adegoke BO, Adjei DN (2011) Effects of aerobic exercise on selected physiological parameters and quality of life in patients with type 2 diabetes mellitus. Int J Gen Med 4:723-727

31. Cheung NW, Cinnadaio N, Russo M, Marek S (2009) A pilot randomised controlled trial of resistance exercise bands in the management of sedentary subjects with type 2 diabetes. Diabetes Res Clin Pract 83:e68-e71

32. Ferrer-Garcia JC, Sanchez Lopez P, Pablos-Abella C et al (2011) Benefits of a home-based physical exercise program in elderly subjects with type 2 diabetes mellitus. Endocrinol Nutr 58:387394, Article in Spanish

33. Fritz T, Caidahl K, Osler M, Ostenson CG, Zierath JR, Wandell P (2011) Effects of Nordic walking on health-related quality of life in overweight individuals with type 2 diabetes mellitus, impaired or normal glucose tolerance. Diabetic Med 28:1362-1372

34. Gram B, Christensen R, Christiansen C, Gram J (2010) Effects of Nordic walking and exercise in type 2 diabetes mellitus: a randomized controlled trial. Clin J Sport Med 20:355-361

35. Kaplan RM, Hartwell SL, Wilson DK, Wallace JP (1987) Effects of diet and exercise interventions on control and quality of life in noninsulin-dependent diabetes mellitus. J Gen Intern Med 2:220-228

36. Krousel-Wood MA, Berger L, Jiang X, Blonde L, Myers L, Webber L (2008) Does home-based exercise improve body mass index in patients with type 2 diabetes? Results of a feasibility trial. Diabetes Res Clin Pract 79:230-236

37. Lam P, Dennis SM, Diamond TH, Zwar N (2008) Improving glycaemic and BP control in type 2 diabetes. The effectiveness of tai chi. Aust Fam Physician 37:884-887

38. Lambers S, van Laethem C, van Acker K, Calders P (2008) Influence of combined exercise training on indices of obesity, diabetes and cardiovascular risk in type 2 diabetes patients. Clin Rehabil 22:483-492

39. Ligtenberg PC, Godaert GL, Hillenaar EF, Hoekstra JB (1998) Influence of a physical training program on psychological well- being in elderly type 2 diabetes patients. Psychological well-being, physical training, and type 2 diabetes. Diabetes Care 21:21962197

40. Lincoln AK, Shepherd A, Johnson PL, Castaneda-Sceppa C (2011) The impact of resistance exercise training on the mental health of older Puerto Rican adults with type 2 diabetes. J Gerontol B Psychol Sci Soc Sci 66:567-570

41. McKay HG, King D, Eakin EG, Seeley JR, Glasgow RE (2001) The diabetes network internet-based physical activity intervention: a randomized pilot study. Diabetes Care 24:1328-1334

42. Nicolucci A, Balducci S, Cardelli P, Zanuso S, Pugliese G (2011) Improvement of quality of life with supervised exercise training in subjects with type 2 diabetes mellitus. Arch Intern Med 171:19511953

43. Plotnikoff RC, Eves N, Jung M, Sigal RJ, Padwal R, Karunamuni N (2010) Multicomponent, home-based resistance training for obese adults with type 2 diabetes: a randomized controlled trial. International J Obes 34:1733-1741

44. Reid RD, Tulloch HE, Sigal RJ et al (2010) Effects of aerobic exercise, resistance exercise or both, on patient-reported health status and well-being in type 2 diabetes mellitus: a randomised trial. Diabetologia 53:632-640

45. Shenoy S, Guglani R, Sandhu JS (2010) Effectiveness of an aerobic walking program using heart rate monitor and pedometer on the parameters of diabetes control in Asian Indians with type 2 diabetes. Prim Care Diabetes 4:41-45

46. Tessier D, Menard J, Fulop T et al (2000) Effects of aerobic physical exercise in the elderly with type 2 diabetes mellitus. Arch Gerontol Geriatr 31:121-132

47. Tsang T, Orr R, Lam P, Comino EJ, Singh MF (2007) Health benefits of tai chi for older patients with type 2 diabetes: the 'Move It For Diabetes study' - a randomized controlled trial. Clin Interv Aging 2:429-439

48. Davidson MB (2005) SF-36 and diabetes outcome measures. Diabetes Care 28:1536-1537

49. U.K. Prospective Diabetes Group (1999) Quality of life in type 2 diabetic patients is affected by complications but not by intensive policies to improve blood glucose or blood pressure control (UKPDS 37). Diabetes Care 22:1125-1136

50. Quah JH, Luo N, Ng WY, How CH, Tay EG (2011) Health-related quality of life is associated with diabetic complications, but not with short-term diabetic control in primary care. Ann Acad Med Singapore 40:276-286

51. American College of Sports Medicine and the American Diabetes Association (2010) Exercise and type 2 diabetes: American College of Sports Medicine and the American Diabetes Association: joint position statement. Med Sci Sports Exerc 42:2282-2303

52. Rimer J, Dwan K, Lawlor DA et al (2012) Exercise for depression. Cochrane Database Syst Rev 7:CD004366

53. Craft LL, Perna FM (2004) The benefits of exercise for the clinically depressed. Prim Care Companion J Clin Psychiatr 6:104111

54. Gonzalez JS, Safren SA, Delahanty LM et al (2008) Symptoms of depression prospectively predict poorer self-care in patients with type 2 diabetes. Diabet Med 25:1102-1107 